\title{
Simulating Magnetised Plasma with the Versatile Advection Code
}

\author{
Rony Keppens ${ }^{1}$ and Gábor Tóth ${ }^{2}$ \\ 1 FOM-Institute for Plasma-Physics Rijnhuizen, \\ P.O. Box 1207, 3430 BE Nieuwegein, The Netherlands, \\ keppens@rijnh.nl \\ 2 Department of Atomic Physics, Eötvös University, \\ Pázmány Péter sétány 2, Budapest H-1117, Hungary, \\ gtoth@hermes.elte.hu
}

\begin{abstract}
Matter in the universe mainly consists of plasma. The dynamics of plasmas is controlled by magnetic fields. To simulate the evolution of magnetised plasma, we solve the equations of magnetohydrodynamics using the Versatile Advection Code (VAC).

To demonstrate the versatility of VAC, we present calculations of the Rayleigh-Taylor instability, causing a heavy compressible gas to mix into a lighter one underneath, in an external gravitational field. Using a single source code, we can study and compare the development of this instability in two and three spatial dimensions, without and with magnetic fields. The results are visualised and analysed using IDL (Interactive Data Language) and AVS (Advanced Visual Systems).

The example calculations are performed on a Cray J90. VAC also runs on distributed memory architectures, after automatic translation to High Performance Fortran. We present performance and scaling results on a variety of architectures, including Cray T3D, Cray T3E, and IBM SP platforms.
\end{abstract}

\section{Magneto-Hydrodynamics}

The MHD equations describe the behaviour of a perfectly conducting fluid in the presence of a magnetic field. The eight primitive variables are the density $\rho(\mathbf{r}, t)$, the three components of the velocity field $\mathbf{v}(\mathbf{r}, t)$, the thermal pressure $p(\mathbf{r}, t)$, and the three components of the magnetic field $\mathbf{B}(\mathbf{r}, t)$. When written in conservation form, the conservative variables are density $\rho$, momentum $\rho \mathbf{v}$, energy density $\mathcal{E}$, and the magnetic field $\mathbf{B}$. The thermal pressure $p$ is related to the energy density as $p=(\gamma-1)\left(\mathcal{E}-\frac{1}{2} \rho v^{2}-\frac{1}{2} B^{2}\right)$, with $\gamma$ the ratio of specific heats. The eight non-linear partial differential equations express: (1) mass conservation; (2) the momentum evolution (including the Lorentz force); (3) energy conservation; and (4) the evolution of the magnetic field in an induction equation. The equations are given by

$$
\frac{\partial \rho}{\partial t}+\nabla \cdot(\rho \mathbf{v})=0
$$




$$
\begin{gathered}
\frac{\partial(\rho \mathbf{v})}{\partial t}+\nabla \cdot\left[\rho \mathbf{v v}+p_{t o t} I-\mathbf{B B}\right]=\rho \mathbf{g} \\
\frac{\partial \mathcal{E}}{\partial t}+\nabla \cdot(\mathcal{E} \mathbf{v})+\nabla \cdot\left(p_{t o t} \mathbf{v}\right)-\nabla \cdot(\mathbf{v} \cdot \mathbf{B B})=\rho \mathbf{g} \cdot \mathbf{v}+\nabla \cdot[\mathbf{B} \times \eta(\nabla \times \mathbf{B})], \\
\frac{\partial \mathbf{B}}{\partial t}+\nabla \cdot(\mathbf{v B}-\mathbf{B v})=-\nabla \times[\eta(\nabla \times \mathbf{B})] .
\end{gathered}
$$

We introduced $p_{t o t}=p+\frac{1}{2} B^{2}$ as the total pressure, $I$ as the identity tensor, $\mathrm{g}$ as the external gravitational field, and defined magnetic units such that the magnetic permeability is unity.

Ideal MHD corresponds to a zero resistivity $\eta$ and ensures that magnetic flux is conserved. In resistive MHD, field lines can reconnect. An extra constraint arises from the non-existence of magnetic monopoles, expressed by $\nabla \cdot \mathbf{B}=0$. The ideal MHD equations allow for Alfvén and magneto-acoustic wave modes, while the induction equation prescribes that flow across the magnetic field entrails the field lines, so that field lines are 'frozen-in'. The field may, in turn, confine the plasma. The MHD description can be used to study both laboratory and astrophysical plasma phenomena. We refer the interested reader to [2] for a derivation of the MHD equations starting from a kinetic description of the plasma, while excellent treatments of MHD theory can be found in, e.g. [4,1].

\section{The Versatile Advection Code}

The Versatile Advection Code (VAC) is a general purpose software package for solving a conservative system of hyperbolic partial differential equations with additional non-hyperbolic source terms $[13,14]$, in particular the hydrodynamic $(\mathbf{B}=0)$ and magnetohydrodynamic equations (1)-(4), with optional terms for gravity, viscosity, thermal conduction, and resistivity.

VAC is implemented in a modular way, which ensures its capacity to model several systems of conservation laws, and makes it possible to share solution algorithms among all systems. A variety of spatial and temporal discretizations are implemented for solving such systems on a finite volume structured grid. The spatial discretizations include two Flux Corrected Transport variants and four Total Variation Diminishing (TVD) algorithms (see [18]). These numerical schemes are shock-capturing and second order accurate in space and time.

Explicit time integration may exploit predictor-corrector and Runge-Kutta time stepping, while for multi-timescale problems, mixed implicit/explicit time integration is available to treat only some variables, or some terms in the governing equations implicitly $[10,11]$. Fully implicit time integration can be of interest when modelling steady-state problems. Typical astrophysical applications where semi-implicit and implicit methods are efficiently used can be found in $[17,9]$.

VAC runs on personal computers (Pentium PC under Linux), on a variety of workstations (DEC, Sun, HP, IBM) and has been used on SGI Power Challenge, Cray J90 and Cray C90 platforms. To run VAC on distributed memory architectures, an automatic translation to High Performance Fortran (HPF) is done 
at the preprocessing phase (see [12]). We have tested the generated HPF code on several platforms, including a cluster of Sun workstations, a Cray T3D, a 16-node Connection Machine 5 (using an automatic translation to CM-Fortran), an IBM SP and a Cray T3E. Scaling and performance is discussed in section 3.

On-line manual pages, general visualisation macros (for IDL, MatLab and $\mathrm{SM}$ ), and file format transformation programs (for AVS, DX, and Gnuplot) facilitate the use of the code and aid in the subsequent data analysis.

In this manuscript, we present calculations done in two and three spatial dimensions, for both hydrodynamic and magnetohydrodynamic problems. This serves to show how VAC allows a single problem setup to be studied under various physical conditions. We have used IDL and AVS to analyse the application presented here. Our data analysis and visualisation encompasses X-term animation, generating MPEG-movies, and video production.

\section{Scaling Results}

As detailed in [12], the source code uses a limited subset of the Fortran 90 language, extended with the HPF forall statement and the Loop Annotation SYntax (LASY) which provides a dimension independent notation. The LASY notation [15] is translated by the VAC preprocessor according to the dimensionality of the problem. Further translation to HPF involves distributing all global non-static arrays across the processors, which is accomplished in the preprocessing stage by another Perl script.

Figure 1 summaries timing results obtained on two vector (Cray J90 and C90) and three massively parallel platforms (Cray T3D, T3E and IBM SP). We solve the shallow water equations (1)-(2) with $\mathbf{B}=0$ and $p=(g / 2) \rho^{2}$ on a $104 \times 104$ grid on $1,2,4,8$, and 13 processors. This simple model problem is described in [16], and our solution method contains the full complexity of a real physics application. We used an explicit TVD scheme exploiting a Roe-type approximate Riemann solver. We plot the number of physical grid cell updates per second against the number of processors (solid lines). The dashed lines show the improved scaling for a larger problem of size $208 \times 208$, up to 16 processors. On all parallel platforms, we exploited the Portland Group pghpf compiler. We find an almost linear speedup on the Cray T3D and T3E architectures, which is rather encouraging for such small problem sizes. Note how the single node execution on the IBM SP platform is a factor of 2 to 3 faster than the Cray T3E, but the scaling results are poor. The figure indicates clearly that for this hydrodynamic application, on the order of 10 processors of the Cray T3E and IBM SP are needed to outperform a vectorised Fortran 90 run on one processor of the Cray C90. Detailed optimisation strategies for all architectures shown in Figure 1 (note the Pentium PC result and the DEC Alpha workstation timing in the bottom left corner) are discussed in [16]. 


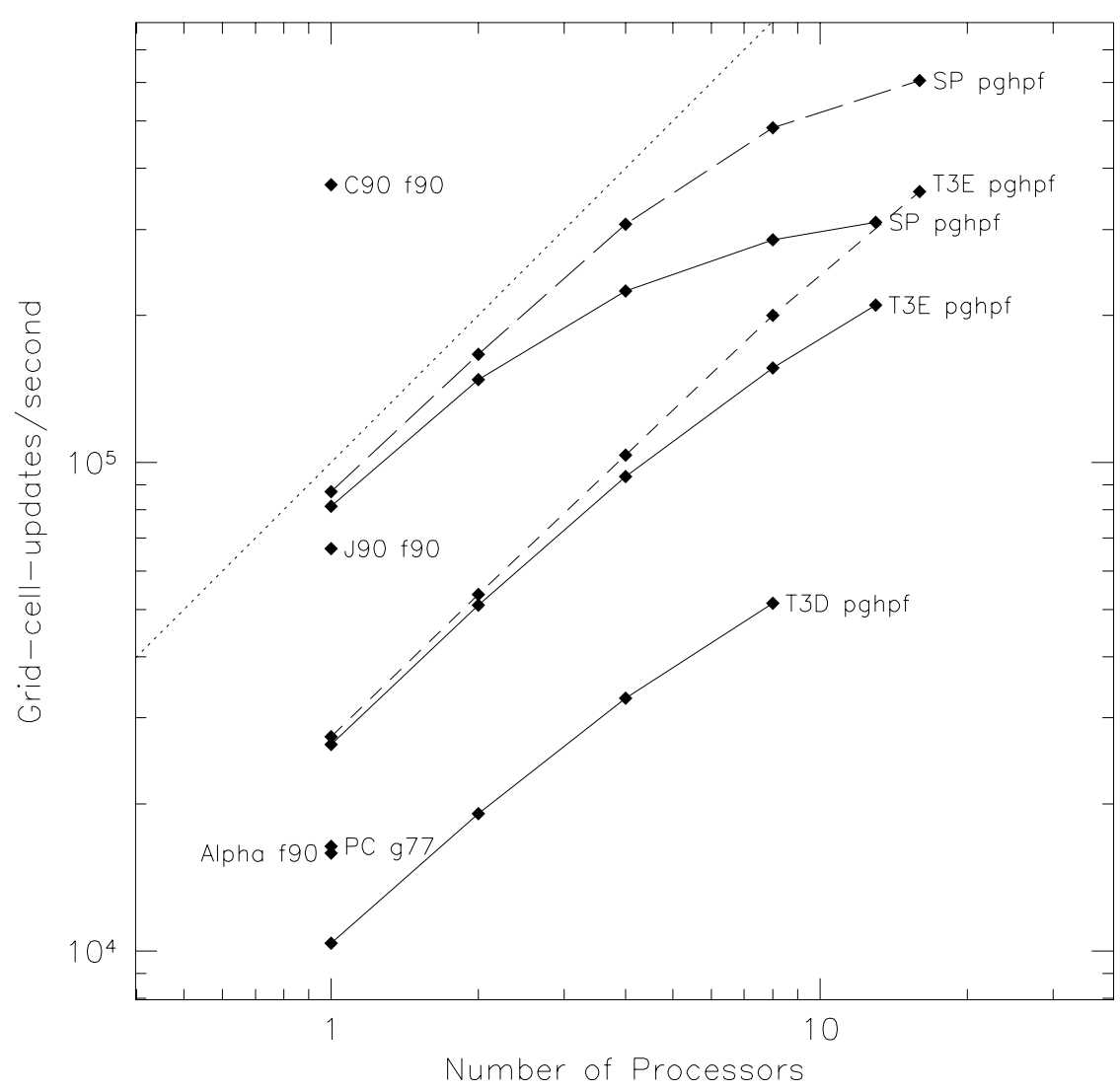

Fig. 1. Combined performance and scaling results for running the Versatile Advection Code on vector and parallel platforms. See text for details. 


\section{Simulating Rayleigh-Taylor Instabilities}

To demonstrate the advantages of having a versatile source code for simulating fluid flow, we consider what happens when a heavy compressible plasma is sitting on top of a lighter plasma in an external gravitational field. Such a situation is unstable as soon as the interface between the two is perturbed from perfect flatness. The instability is known as the Rayleigh-Taylor instability. Early analytic investigations date back to a comprehensive and detailed analysis given by Chandrasekhar [3].

The initial configuration is one where two layers of prescribed density ratio (dense to light ratio of $\rho_{d} / \rho_{l}=10$ ) are left to evolve between two planes $(y=0$ and $y=1)$, with gravity pointing downwards $\left(\mathrm{g}=-\hat{e}_{y}\right.$ unit vector). The heavy plasma on the top is separated from the light plasma below it by the surface $y=y_{0}+\epsilon \sin \left(k_{x} x\right) \sin \left(k_{z} z\right)$. Initially, both are at rest with $\mathbf{v}=0$, and the thermal pressure is set according to the hydrostatic balance equation (cantered differenced formula $d p / d y=-\rho$ ). Boundary conditions make top and bottom perfectly conducting solid walls, while the horizontal directions are periodic. We then exploit the options available in VAC to see how the evolution changes when going from two to three spatial dimensions, and what happens when magnetic fields are taken along. All calculations are done on a Cray J90, where we preprocess the code to Fortran 90 for single-node execution.

\subsection{Two-dimensional Simulations}

Figure 2 shows the evolution of the density in two two-dimensional simulations without and with an initial horizontal magnetic field $\mathbf{B}=0.1 \hat{e}_{x}$. Both simulations are done on a uniform $100 \times 100$ square grid, and the parameters for the initial separating surface are $y_{0}=0.8, \epsilon=0.05$, and $k_{x}=2 \pi$ (there is no $z$ dependence in $2 \mathrm{D})$. The data is readily analysed using IDL.

In both cases, the heavy plasma is redistributed in falling spikes or pillars, also termed Rayleigh-Taylor 'fingers', pushing the lighter plasma aside with pressure building up underneath the pillars. However, in the ideal MHD case, the frozen-in field lines are forced to move with the sinking material, so it gets wrapped around the pillars. The extra magnetic pressure and tension forces thereby confine the falling dense plasma and slow down the sinking and mixing process. In fact, since we took the initial displacement perpendicular to the horizontal magnetic field, we effectively maximised its stabilising influence.

In [3], the linear phase of the Rayleigh-Taylor instability in both hydrodynamic and magnetohydrodynamic incompressible fluids is treated analytically. The stabilising effect of the uniform horizontal magnetic field is evident from the expression of the growthrate $n$ as a function of the wavenumber $k_{x}$

$$
n^{2}=g k_{x} \frac{\rho_{d}-\rho_{l}}{\rho_{d}+\rho_{l}}-\frac{B^{2} k_{x}^{2}}{2 \pi\left(\rho_{d}+\rho_{l}\right)} .
$$

Hence, while the shortest wavelength perturbations are the most unstable ones in hydrodynamics $(B=0)$, all wavelengths below a critical $\lambda_{\text {crit }}=B^{2} / g\left(\rho_{d}-\rho_{l}\right)$ 


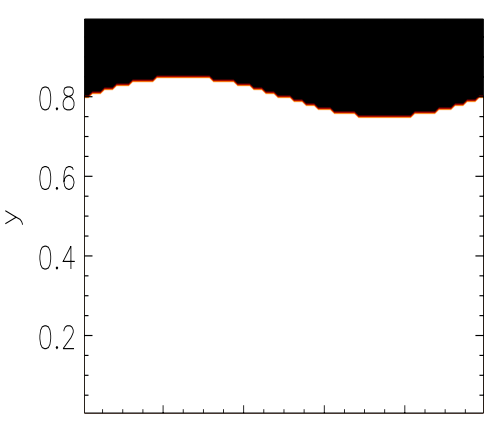

$\begin{array}{llll}0.2 & 0.4 & 0.6 & 0.8\end{array}$

time $=0.0000$

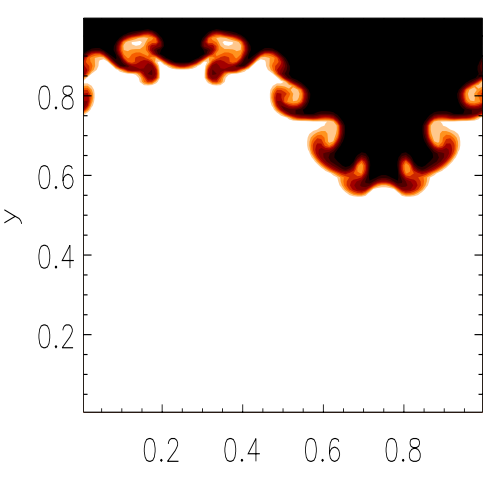

time $=1.0040$

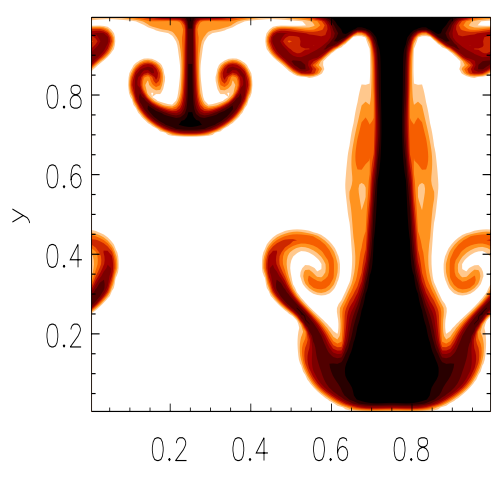

time $=2.0074$

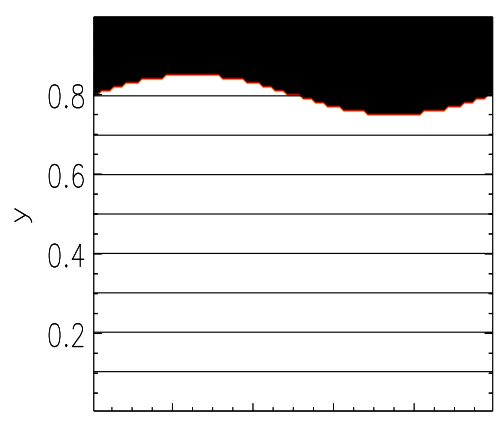

$\begin{array}{llll}0.2 & 0.4 & 0.6 & 0.8\end{array}$

time $=0.0000$

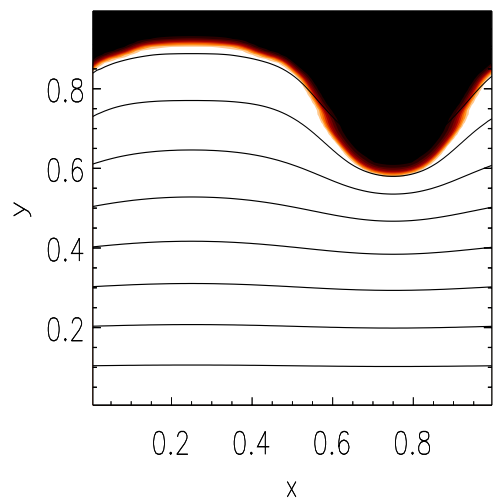

time $=1.0032$

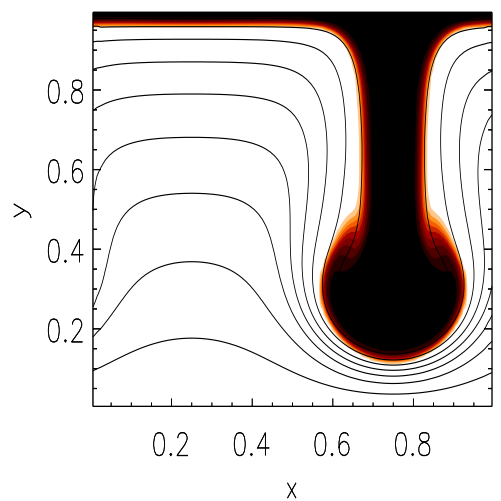

time $=2.0005$

Fig. 2. Rayleigh-Taylor instability simulated in two spatial dimensions, in a hydrodynamic (left) and magnetohydrodynamic (right) case. The logarithm of the density and, in the magnetohydrodynamic case, also the magnetic field lines, are plotted. 
are effectively suppressed by a horizontal magnetic field of strength $B$. Similarly, our initial perturbation with $\lambda=2 \pi / k_{x}=1$ will be stabilised as soon as the magnetic field surpasses a critical field strength $B_{\text {crit }}=\sqrt{g \lambda\left(\rho_{d}-\rho_{l}\right)} \simeq 0.95$.

The simulations confirm and extend these analytic findings: the predicted growth-rate can be checked (noting that our simulations are compressible), while the further non-linear evolution can be investigated. The discrete representation of the initial separating surface causes intricate small-scale structure to develop in the simulation at left of Figure 2. This is consistent with the fact that in a pure hydrodynamic case, the shortest wavelengths are the most unstable ones. Naturally, the simulation is influenced by numerical diffusion, while the periodic boundary conditions and the initial state select preferred wavenumbers. The suppression of short wavelength disturbances in the MHD case is immediately apparent, since no small-scale structure develops. The simulation at right has an initial plasma beta (ratio of gas to magnetic pressure forces) of about 400 . For higher plasma beta yet, the MHD case will resemble the hydrodynamic simulation more closely, while a stronger magnetic field $\left(\mathbf{B}=\hat{e}_{x}\right)$ suppresses the development of the instability entirely, as theory predicts.

Note also how the falling pillars develop a mushroom shape (left frames) as a result of another type of instability caused by the velocity shear across their edge: the Kelvin-Helmholtz instability $[3,7,8]$. The lighter material is swept up in swirling patterns around the sinking spikes. In the MHD simulation (right frames) the Kelvin-Helmholtz instability does not develop due to the stabilising effect of the magnetic field. Typically however, both instabilities play a crucial role in various astrophysical situations. Two dimensional MHD simulations of Rayleigh-Taylor instabilities in young supernova remnants [5] demonstrate this, and confirm the basic effects evident from Figure 2: magnetic fields get warped and amplified around the 'fingers'. General discussions of these and other hydrodynamic and magnetohydrodynamic instabilities are found in [3].

\subsection{Three-dimensional Simulations}

In Figure 3, we present a snapshot of a hydrodynamical calculation in a 3D $50 \times 50 \times 50$ unit box, where the initial configuration has both $k_{x}=2 \pi$ and $k_{z}=$ $2 \pi$. With gravity downwards, we look into the box from below. On two vertical cuts, we show at time $t=2$ (i) the logarithm of the density in a colour scale and (ii) the streamlines of the velocity field, coloured according to the (logarithm of the) density. The cuts are chosen to intersect the initial separating surface between the heavy and the light plasma at its extremal positions where the motion is practically two-dimensional. 3D effects are readily identified by direct comparison with the two-dimensional hydrodynamic calculation. The time series of the 3D data set has been analysed using AVS (a video is made with AVS to demonstrate how density, pressure and velocity fields evolve during the mixing process).

Figure 4 shows the evolution of a three-dimensional MHD calculation at times $t=1$ and $t=2$. We show an iso-surface of the density (at $1 \%$ above the initial value for $\rho_{d}$ ), coloured according to the thermal pressure. A cutting plane also 


\section{$\log (\mathbf{R h o}) \&$ Streamlines}

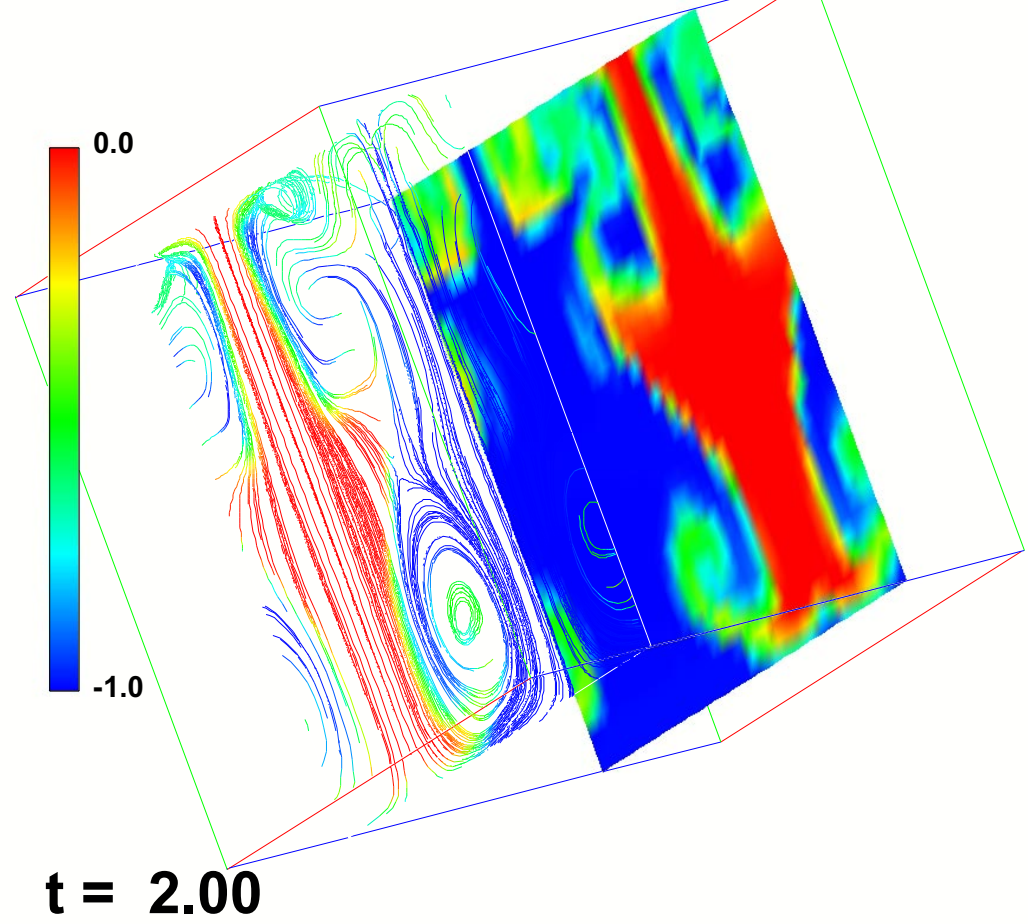

Fig. 3. Rayleigh-Taylor instability in 3D, purely hydrodynamic. We show streamlines (left) and density contours (right) in two vertical cutting planes. 
shows the vertical stratification of the thermal pressure. Note the change in the initial configuration $\left(k_{x}=6 \pi\right.$ and $k_{z}=4 \pi$, with $\left.y_{0}=0.7\right)$ : more and narrower spikes are seen to grow and to split up. The AVS analysis of the full time series shows how droplets form at the tips of the falling pillars, which seem to expand horizontally to a critical size before continuing their fall. At the same time, the magnetic field gets wrapped around the falling pillars. Figure 4 nicely confirms that places where spikes branch into narrower ones correspond to places with excess pressure underneath. Similar studies of incompressible 3D ideal MHD cases are found in [6]. They confirm that strong tangential fields suppress the growth as expected from theoretical considerations, while the Rayleigh-Taylor instability acts to amplify magnetic fields locally. In such magnetic fluids, parameter regimes exist where secondary Kelvin-Helmholtz instabilities develop, just as in the hydrodynamic situation of Figure 3 (note the regions of strong vorticity in the streamlines).

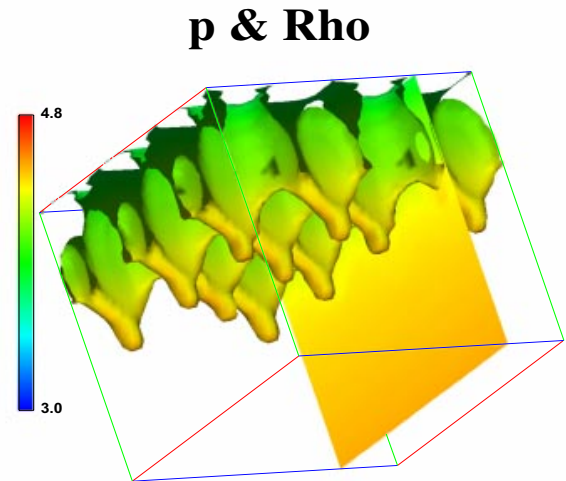

$t=1.00$

$\rho: 1.01$

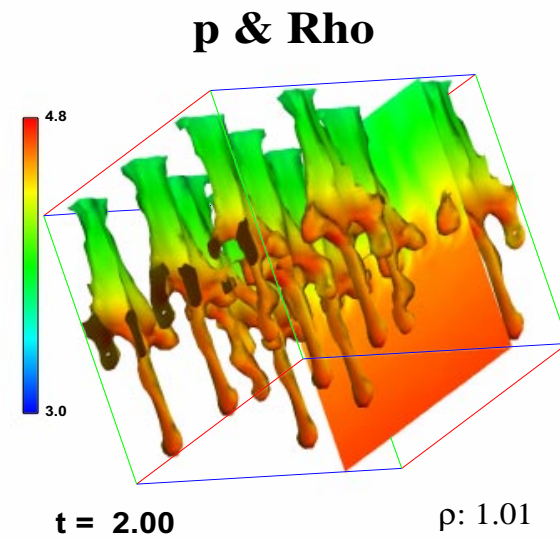

Fig. 4. 3D MHD Rayleigh-Taylor instability. At two consecutive times, an isosurface of the density is coloured according to the thermal pressure. The thermal pressure is also shown in a vertical cut.

\section{Conclusions}

We have developed a powerful tool to simulate magnetised fluid dynamics. The Versatile Advection Code runs on many platforms, from PC's to supercomputers including distributed memory architectures. The rapidly maturing HPF compilers can yield scalable parallel performance for general fluid dynamical simulations. Clearly, the scaling and performance of VAC make high resolution 3D simulations possible, and detailed investigations may broaden our insight in the intricate dynamics of magneto-fluids and plasmas. 
We presented simulations of the Rayleigh-Taylor instability in two and three spatial dimensions, with and without magnetic fields. VAC allows one to do all these simulations with a single problem setup, since the equations to solve and the dimensionality of the problem is simply specified in a preprocessing phase. Data analysis can be done using a variety of data visualisation packages, including IDL and AVS as demonstrated here. In the future, we plan to use VAC to investigate challenging astrophysical problems, like winds and jets emanating from stellar objects [9], magnetic loop dynamics, accretion onto black holes, etc. .

Web-site info on the code is available at http://www.phys.uu.nl/ ${ }^{\text {toth/ }}$ and at http://www . phys.uu.nl/ $\mathrm{mpr} /$. MPEG-animations of various test problems can also be found there.

\section{Acknowledgements}

The Versatile Advection Code was developed as part of the project on 'Parallel Computational Magneto-Fluid Dynamics', funded by the Dutch Science Foundation (NWO) Priority Program on Massively Parallel Computing, and coordinated by Prof. Dr. J.P. Goedbloed. Computer time on the CM-5, the Cray T3E and IBM SP machines was sponsored by the Dutch 'Stichting Nationale Computerfaciliteiten' (NCF). R.K. performed the simulations on the Cray T3D, J90, and Sun workstation cluster at the Edinburgh Parallel Computing Centre with support from the TRACS programme as part of his research at FOM. G.T. receives a postdoctoral fellowship (D 25519) from the Hungarian Science Foundation (OTKA), and is supported by the OTKA grant F 017313.

\section{References}

1. Biskamp, D.: Nonlinear Magnetohydrodynamics. Cambridge Monographs on Plasma Physics 1, Cambridge University Press, Cambridge (1993) 681

2. Bittencourt, J.A.: Fundamentals of Plasma Physics. Pergamon Press, Oxford (1986) 681

3. Chandrasekhar, S.: Hydrodynamic and Hydromagnetic stability. Oxford University Press, New York (1961) 684, 684, 686, 686

4. Freidberg, J.P.: Ideal Magnetohydrodynamics. Plenum Press, New York (1987) 681

5. Jun, B.-I., Norman, M.L.: MHD simulations of Rayleigh-Taylor instability in young supernova remnants. Astrophys. and Space Science 233 (1995) 267-272 686

6. Jun, B.-I., Norman, M.L., Stone, J.M.: A numerical study of Rayleigh-Taylor instability in magnetic fluids. Astrophys. J. 453 (1995) 332-349 688

7. Keppens, R., Tóth, G., Westermann, R.H.J., Goedbloed, J.P.: Growth and saturation of the Kelvin-Helmholtz instability with parallel and anti-parallel magnetic fields. Accepted by J. Plasma Phys. (1998) 686

8. Keppens, R., Tóth, G.: Non-linear dynamics of Kelvin-Helmholtz unstable magnetized jets: three-dimensional effects. Submitted for publication (1998) 686

9. Keppens, R., Goedbloed, J.P.: Numerical simulations of stellar winds: polytropic models. Accepted by Astron. \& Astrophys. (1998) 681, 689 
10. Keppens, R., Tóth, G., Botchev, M.A., van der Ploeg, A.: Implicit and semi-implicit schemes in the Versatile Advection Code: algorithms. Submitted for publication (1998) 681

11. van der Ploeg, A., Keppens, R., Tóth, G.: Block Incomplete LU-preconditioners for Implicit Solution of Advection Dominated Problems. In: Hertzberger, B., Sloot, P. (eds.): Proceedings of High Performance Computing and Networking Europe 1997. Lecture Notes in Computer Science, Vol. 1225. Springer-Verlag, Berlin Heidelberg New York (1997) 421-430 681

12. Tóth, G.: Preprocessor based implementation of the Versatile Advection Code for workstations, vector and parallel computers. Proceedings of VECPAR'98 (3rd international meeting on vector and parallel processing), June 21-23 (1998), Porto, Portugal, Part II, p. 553-560. 682, 682

13. Tóth, G.: Versatile Advection Code. In: Hertzberger, B., Sloot, P. (eds.): Proceedings of High Performance Computing and Networking Europe 1997. Lecture Notes in Computer Science, Vol. 1225. Springer-Verlag, Berlin Heidelberg New York (1997) 253-262 681

14. Tóth, G.: A general code for modeling MHD flows on parallel computers: Versatile advection code. Astrophys. Lett. \& Comm. 34 (1996) 245681

15. Tóth, G.: The LASY Preprocessor and Its Application to general Multidimensional Codes. J. Comput. Phys. 138 (1997) 981-990 682

16. Tóth, G., Keppens, R.: Comparison of Different Computer Platforms for Running the Versatile Advection Code. In: Sloot, P., Bubak, M., and Hertzberger, B. (eds.): Proceedings of High Performance Computing and Networking Europe 1998, Lecture Notes in Computer Science, Vol. 1401, Springer-Verlag, Berlin Heidelberg New York (1998) 368-376 682, 682

17. Tóth, G., Keppens, R., Botchev, M.A.: Implicit and semi-implicit schemes in the Versatile Advection Code: numerical tests. Astron. \& Astrophys. 332 (1998) 11591170681

18. Tóth, G., Odstrčil, D.: Comparison of some Flux Corrected Transport and Total Variation Diminishing Numerical Schemes for Hydrodynamic and Magnetohydrodynamic Problems. J. Comput. Phys. 128 (1996) 82681 